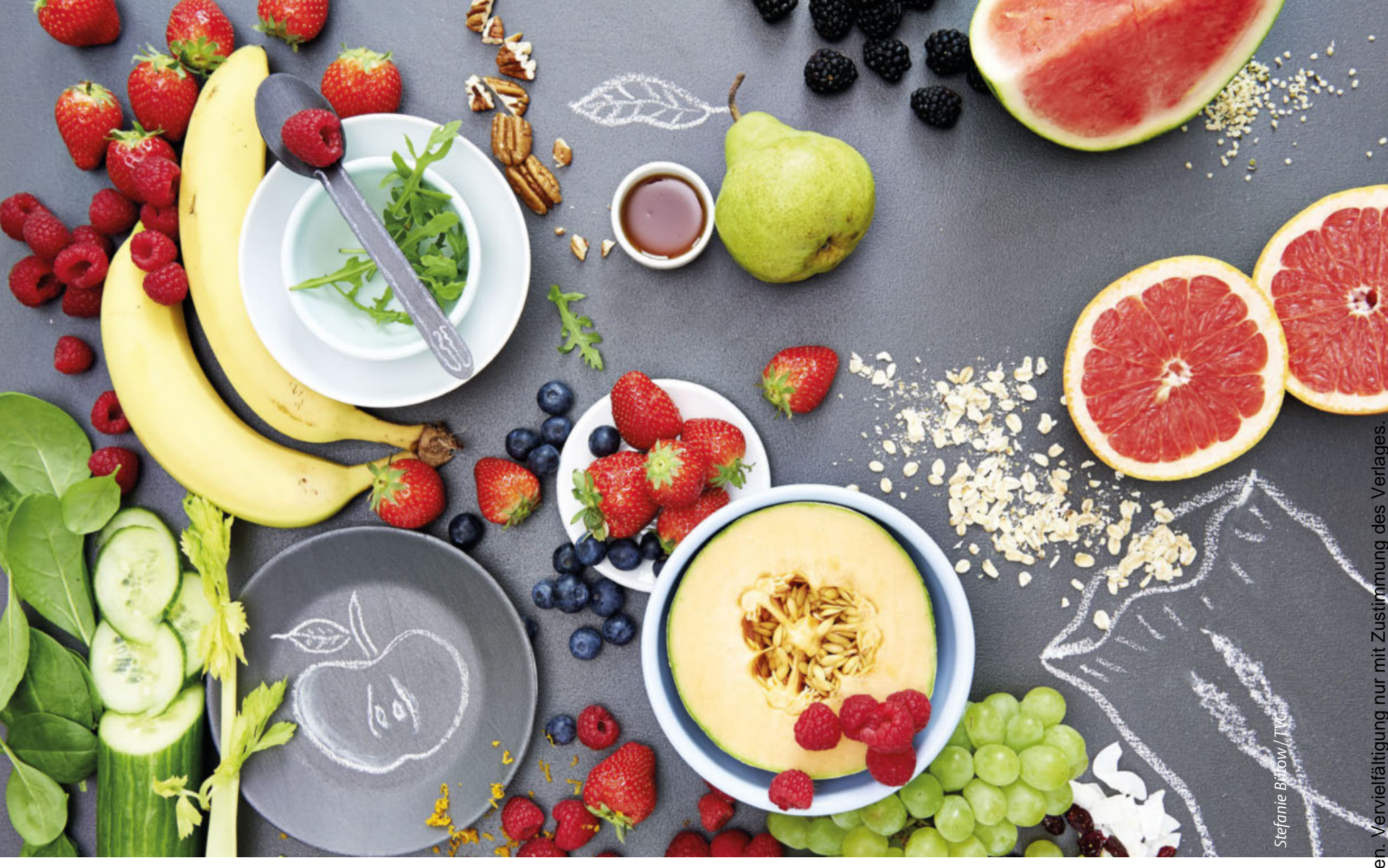

\title{
Parodontitis: Mit der Ernährung die Therapie
} unterstützen

Reichlich Antioxidantien, entzündungshemmende Fettsäuren und Mineralien

Die Mundhöhle und die Ernährung stehen in einer wechselseitigen Beziehung: Einerseits ist ein gesunder Kauapparat eine wichtige Voraussetzung für die Nahrungsaufnahme, auf der anderen Seite sind die Zähne, Kieferknochen und Schleimhäute auf eine gute Vitalstoffversorgung angewiesen. Durch den Kontakt mit der Außenwelt und die dadurch bedingte Konfrontation mit Mikroorganismen läuft in der Mundhöhle die Abwehr auf Hochtouren. Schwächelt das Immunsystem, zeigt sich das rasch in Form von entzündetem Zahnfleisch oder Pilzinfektionen (z.B. Soor). Ebenso werden bestimmte Nährstoffmängel sichtbar, das wohl bekann- teste Beispiel ist der Skorbut (Vitamin-CMangel). Wird also die Vitalstoffzufuhr optimiert, erhöht sich die Widerstandskraft der oralen Gewebe. So ergänzt eine ausgewogene Ernährung, die sich an der mediterranen Kost orientiert, Ihre Mundhygienemaßnahmen sinnvoll.

\section{Vitamin C und Folsäure}

Frisches Gemüse und Obst wirkt in unserem Körper basisch. Hinzu kommt ein hoher Anteil an Pflanzenstoffen, die antioxidative, entzündungshemmende und abwehrstärkende Eigenschaften entfalten. Unter den Vitaminen spielen im Zusammenhang zur
Parodontitis besonders Vitamin C und Folsäure eine Rolle. Früher fielen den Seefahrern die Zähne aus, weil Vitamin $C$ in der kargen Seemannskost fehlte. Erst als man Sauerkraut und Zitronen mit an Bord nahm, verlor der Skorbut seinen Schrecken. Vitamin C unterstützt die lokale Abwehr, die Bindegewebsbildung und damit die Regeneration des Zahnfleischs. Da Vitamin C hitzeempfindlich ist und während des Garprozesses verlorengeht, muss Frisches verzehrt werden. Besonders reich an Vitamin C sind: - Sanddornsaft

- Schwarze Johannisbeeren

- Petersilie 
- Paprika

- Brennnesselblätter

- Kiwi

- Gemüsefenchel

- Papaya

Als natürliche, Vitamin-C-reiche Nahrungsergänzungsmittel bieten sich AcerolaLutschtabletten, Hagebutten- und Brennnesselpulver an.

Die Mundschleimhaut erneuert sich regelmäßig durch Zellteilung. Dafür wird u.a. Folsäure benötigt. Ein Folsäuremangel kann sich in entzündetem Zahnfleisch, Zahnfleischbluten und einer wunden Zunge zeigen. Wird die Folsäureaufnahme optimiert, regenerieren sich die Schleimhäute. Gute Folsäurequellen sind frische Sojasprossen, Blattsalate, Petersilie, grüne Bohnen, Hülsenfrüchte und Nüsse. Als natürliche Nahrungsergänzung bietet sich Gerstengraspulver an.

\section{Hochwertige Fette und Öle mit Omega-3-Fettsäuren}

Die Auswertung einer Gesundheitsstudie (National Health and Nutrition Examination Survey = NHANES, 1999-2004) hatte ergeben, dass Erwachsene, die vermehrt Omega-3-Fettsäuren über die tägliche Ernährung aufnehmen, seltener eine Parodontitis entwickeln. Diese Beobachtung lässt sich auf die entzündungshemmenden Eigenschaften der Omega-3-Fettsäuren zurückführen. Innerhalb der Omega-3-Fettsäuren werden die $\alpha$-Linolensäure, die Eicosapentaensäure (EPA) und die Docosahexaensäure (DHA) unterschieden. Während $\alpha$-Linolensäure in pflanzlichen Nahrungsmitteln vorkommt, finden sich die beiden anderen vor allem in tierischen Speisen wie Lachs, Makrele, Heilbutt, Hering und Thunfisch, aber auch in Eiern, Milch- und Fleischfett, wobei die Fütterung der Tiere den Gehalt beeinflusst. Beispielsweise enthält das Milchfett von Weidekühen bzw. Milchkühen mit Grünfutter-, Heu- und Grassilagefütterung deutlich mehr Omega-3-Fettsäuren, als bei konventioneller Fütterung mit Mais und Kraftfutter. Für die Versorgung mit $\alpha$-Linolensäure sind besonders Walnussöl, Leinöl, Rapsöl, Hanföl und Chiasamenöl zu empfehlen.

\section{Kalzium und Vitamin D}

Kalzium erfüllt für den Zahnhalteapparat (lat.: Parodont) gleich mehrere Funktionen. Einerseits ist es Bestandteil der Zahnhartund Knochensubstanz, andererseits unter- stützt es das Immunsystem. Laut einer Gesundheitsstudie steigt bei einer zu geringen Kalziumaufnahme (unter 500 mg pro Tag) das Risiko für eine Parodontitis an. Zusätzlich hängt die Kalziumfunktion von einer guten Vitamin-D-Versorgung ab. An Sonnentagen bildet der Körper Vitamin D in der Haut. Die Ernährungsgesellschaften weisen darauf hin, dass in Deutschland die körpereigene Vitamin-D-Bildung vermutlich nur von April bis September den Bedarf decken kann, sodass von Oktober bis März auf eine ausreichende orale Zufuhr geachtet werden sollte. Zur Sicherheit kann der Vitamin-DStatus im Blut bestimmt werden. Vitamin D ist in fettreichen Fischen enthalten. Danach folgen mit deutlich geringeren Mengen Leber, Eigelb, Milchprodukte und Champignons. Bei nachgewiesenem Vitamin-DMangel wird meist die Einnahme eines Nahrungsergänzungsmittels notwendig.

Für die Optimierung der Kalziumzufuhr eignen sich:

- Emmentaler Käse, Naturjoghurt, Kefir

- grünes Gemüse wie Broccoli, Grünkohl und Fenchel

- Körnerfrüchte und Nüsse wie Amaranth, Quinoa, Hirse, Sesam, Mandeln und Mohn

- Wildkräuter wie Brennnesseln, Schnittlauch und Rauke

Durch regelmäßige Bewegung und Sport wird der Knochenstoffwechsel angeregt, sodass Kalzium besser in die Knochen einlagert werden kann.

\section{Frisch, regional, mediterran ...}

Zur Orientierung bietet sich die traditionelle mediterrane Kost an. Sie zeichnet sich durch folgende Merkmale aus:

- hoher Anteil an pflanzlichen Nahrungsmitteln lokalen Ursprungs in roher oder gering verarbeiteter Form (Gemüse, Obst, Hülsenfrüchte, Nüsse, Körner- und Getreideprodukte)

- geringer bis mäßiger Anteil tierischer Nahrungsmittel (Käse und Joghurt täglich in geringen Mengen, Fisch und Geflügel mehrmals pro Woche, rotes Fleisch, Wurstwaren, Eier mehrmals pro Monat)

- Olivenöl als Hauptfettquelle (ersetzt Butter und Margarine)

- reichliche Verwendung von frischen Kräutern und Gewürzen (Basilikum, Oregano, Thymian, Knoblauch, Zwiebeln, Salbei u.a.)
- geringer bis mäßiger Weinkonsum (vor allem Rotwein zu den Mahlzeiten) - es wird selbst gebacken und gekocht Die aromatischen Rezepte der mediterranen Küche bringen nicht nur Genuss, sondern nützen Ihrer Gesundheit.

Dr. Henrike März, Ernährungswissenschaftlerin, Markt Rettenbach

Online zu finden unter

http://dx.doi.org/10.1055/a-0629-6991

Anmerkungen Ihres Arztes

Mit den besten Grüßen überreicht durch: 